Results At intervention conclusion, children's objectively measured physical activity was similar across groups. Maternal beliefs about television and diet and corresponding child behaviours were more favourable in intervention than control participants. For example, fewer intervention group mothers believed television is helpful for development (67\% vs $43 \%$ ), and parents should offer other foods if their child doesn't eat their meal ( $44 \%$ vs $20 \%$; both $\mathrm{p}<0.001)$. More intervention mothers believed parents should include fruit or vegetables in all children's meals and snacks ( $96 \%$ vs $91 \%, p=0.04)$, and that television should be turned off when children are eating $(90 \%$ vs $81 \%, p=0.02$ ). Compared to control group infants, those in the intervention group were significantly more likely to consume two or more serves of fruit ( $81 \%$ vs $70 \%, p=0.03$ ), vegetables $(56 \%$ vs $42 \%$, $\mathrm{p}=0.04)$ and both fruit and vegetables daily (39\% vs $26 \%, \mathrm{p}<0.001)$ and watched an average of $92 \mathrm{~min}$ less television per week ( $\beta=-13.8,95 \%$ CI -26.4 to -1.2$)$.

Conclusions The Melbourne InFANT Program, a low-dose, low-cost obesity prevention intervention, shows promising impact on parent attitudes and beliefs and on children's diet and television viewing behaviours but not physical activity.

\section{$01-5.2$ MEAT, FISH AND ESOPHAGEAL CANCER RISK: A SYSTEMATIC REVIEW AND META-ANALYSIS}

doi:10.1136/jech.2011.142976a.37

${ }^{1} \mathrm{M}$ Salehi, ${ }^{* 2} \mathrm{~F}$ Kolahdooz, ${ }^{1} \mathrm{M}$ Moradi, ${ }^{3} \mathrm{M}$ H Salehi, ${ }^{1} \mathrm{M}$ Nojomi. ${ }^{1}$ Tehran University of Medical Sciences, Tehran, Iran; ${ }^{2}$ Food \& Drug Division, Ministry of Health and Medical Education, Tehran, Iran; ${ }^{3}$ Mashhad University of Medical Sciences, Mashhad, Iran

Introduction Esophageal cancer is the 8th most common cancer and the 6 th leading cause of cancer mortality worldwide. Mortality from this cancer is high and despite improvements in treatment, the 5 year survival rate still remains low at around $10-13 \%$. The associations between some of risk factors with esophageal cancer are well defined while the role of diet remains controversial. Thus, we sought to examine quantitatively the association between intake of meat and fish and esophageal cancer.

Methods We searched major database for published studies (January 1990 through January 2011) on the associations between consumption of total meat, red meat, processed meat, poultry, and fish and risk of esophageal cancer. Random-effects models were used to pool study results. We conducted subgroup analyses by, histologic subtypes of esophageal cancer, nationality, and potential confounders.

Results We identified three cohort studies and 30 case control studies. The pooled RR of esophageal cancer for intakes of the highest group compared with the lowest group of total meat, red meat, processed meat, poultry and fish were 1.02 (95\% CI 0.89 to 1.18), 1.42 (95\% CI 1.09 to 1.86 ), 1.41 (95\% CI 1.12 to -1.78$), 0.87$ ( $95 \%$ CI 0.60 to 1.24 ), 0.77 (95\% CI 0.62 to 0.97 ) respectively.

Conclusion Our results suggest that high intake of red meat and processed meat may be associated with a higher risk of esophageal cancer, whereas high fish intake may be related to a lower risk.

\section{1-5.3 THE ROLE OF MILK AND DAIRY PRODUCTS IN CHILDHOOD OBESITY: EVIDENCE FROM THE HONG KONG'S “CHILDREN OF 1997" BIRTH COHORT}

doi:10.1136/jech.2011.142976a.38

L L Shi, ${ }^{*}$ M Tarrant, L L Hui, M K Kwok, T H Lam, G M Leung, C M Schooling. The University of Hong Kong, Hong Kong, China

Introduction Observational studies, mainly from western populations, suggest that dairy product consumption is inversely associated with adiposity. However, in such populations, there is a limited range of dairy product intake while dietary intake and obesity share social patterning making evidence from non-western developed settings valuable in distinguishing whether the observed associations are biologically mediated or socially confounded.

Methods We used multivariable linear regression to examine the adjusted association of the frequency of milk or other dairy product consumption at 11 years with clinically measured body mass index (BMI) $z$-scores at about 13 years, relative to the 2007 WHO growth reference in a large $(n=8327)$, population-representative Chinese birth cohort, comprising 88\% of all births in Hong Kong in April and May 1997.

Results Of the original 8327 cohort members, 7933 are alive, participating and living in Hong Kong. At approximately 13 years, 7488 had clinically assessed BMI. Socio-economic position was positively associated with frequency of milk and other dairy product consumption. Neither milk nor other dairy product consumption was associated with BMI z-score (milk $-0.02,95 \% \mathrm{CI}-0.06$ to 0.03 and dairy products $0.03,95 \% \mathrm{CI}-0.01$ to 0.07 ), adjusted for sex, mother's birthplace, highest parental education, pubertal stage, physical activity and other food consumption.

Conclusions In a non-western setting, milk and other dairy product consumption was not associated with adiposity, suggesting that any observed anti-obesigenic effects in western settings may be due to socially patterned confounding by socio-economic position.

\section{1-5.4 FAMILIAL AGGREGATION IN NUTRIENT INTAKE PATTERNS: COMPARING INTERGENERATIONAL AND PRENATAL-POSTNATAL EFFECTS IN LIFEWAYS CROSS GENERATION COHORT STUDY}

doi:10.1136/jech.2011.142976a.39

${ }^{1,2}$ A Shrivastava, ${ }^{* 1,2} \mathrm{C}$ Murrin, ${ }^{1,2} \mathrm{C}$ Kelleher, for the Lifeways Cross Generation Cohort Study Steering Group. ${ }^{1}$ Health Research Board Centre for Diet and Health Research, University College Dublin, Dublin, Ireland; ${ }^{2}$ School of Public Health, Physiotherapy and Population Science, University College Dublin, Dublin, Ireland

Introduction Familial influence on dietary behaviours of children is expressed through genetic predispositions, maternal-environment and home-environment. Disaggregating such effects requires relatively rare cross-generational study designs. This analysis examined associations of maternal, paternal and grandparental dietary intake prenatally; and maternal and maternal-grandmothers dietary intake post-natally with child's dietary intake.

Methods Pre-natal dietary information was available for expectant mothers $(\mathrm{N}=1119)$, fathers $(\mathrm{N}=331)$, maternal-grandmothers $(\mathrm{N}=285)$, maternal-grandfathers $(\mathrm{N}=163)$, paternal-grandmothers $(\mathrm{N}=163)$, and paternal-grandfathers $(\mathrm{N}=96)$ through a validated food frequency questionnaire (FFQ). At 6 year follow-up, when children averaged age 5 , dietary information was re-collected for mothers $(n=558)$ and some maternal-grandmothers $(N=53)$ using the same FFO. A child's FFQ version was used for children $(\mathrm{N}=567)$. Association for energy, macronutrients and fibre intake were compared using Pearson's interclass and intraclass correlations. Nutrients were log transformed and adjusted for energy intake in interclass correlations.

Results Though the correlations were weakly moderate $(r<0.35)$ in strength, a clear pattern emerged. Positive statistically significant correlations were found for energy, macronutrients and fibre intake within children's nuclear families. Correlations were stronger for maternal postnatal-child pairs compared to maternal prenatal-child pairs. The father-child associations were significant (except for fat) but weaker than mother-child associations. Maternal grandmother-mother associations were found for protein, fat and fibre intakes. A significant positive intraclass correlations was observed in nutrient intakes of maternal grandmothers-mothers-child triads, not found in paternal lines.

Conclusion The stronger maternal associations with children's nutrient intake and existence of maternal grandmother-motherchild associations support evidence for maternal-environment programming influences. 